\title{
Volumetric magnetic resonance imaging study of the brain in subjects with sex chromosome aneuploidies
}

Michael M Warwick, Gillian A Doody, Stephen M Lawrie, Julia N Kestelman, Jonathan J K Best, Eve C Johnstone

\begin{abstract}
Objectives-Cognitive impairment has been reported in people with sex chromosome aneuploides (SCAs) and it has been proposed that the presence of an extra sex chromosome may have an adverse effect on neurodevelopment. This study examines the hypothesis with structural MRI of the brain.

Methods-Thirty two subjects with SCA (XXX $(n=12)$, XYY $(n=10)$, and XXY $(n=10))$ from a birth cohort study were matched groupwise for age, parental social class, and height with normal controls (13 female, 26 male). Brain MRI , measurements of IQ, and a structured psychiatric interview were performed.

Results-The XXX females and XXY males had significantly smaller whole brain volumes than controls of the same phenotypic sex $(p=0.003$ and $p \leqslant 0.05$ respectively). The XXY group also had bilaterally enlarged lateral ventricles $(p \leqslant 0.05)$. No significant differences were found between the XYY group and controls. IQ scores in all SCA groups were lower than in the control groups.

Conclusions-The main result of reduced brain volumes in XXX and XXY subjects, but not in XYY subjects, indicates that the presence of a supernumerary $X$ chromosome has a demonstrable effect on brain development.
\end{abstract}

(F Neurol Neurosurg Psychiatry 1999;66:628-632)

Department of

Psychiatry, Royal

Edinburgh Hospital,

Edinburgh, UK

M M Warwick

G A Doody

S M Lawrie

J N Kestelman

J J K Best

E C Johnstone

Magnetic Resonance Imaging Unit, City Hospital, Edinburgh, UK

J J K Best

Correspondence to: Dr MM Warwick, Department of Psychiatry, Royal Edinburgh Hospital, Edinburgh, EH10 5HF, UK.

Received 15 June 1998 and in revised form

16 October 1998

Accepted 6 November 1998
Keywords: sex chromosome aneuploides; magnetic resonance imaging; schizotypy

Over the past 50 years refinement in genetic techniques has enabled the identification and subsequent characterisation of sex chromosome aneuploidies (SCAs). Early studies focused on the phenotypic manifestations of the XYY genotype, in both institutional and general populations. ${ }^{1-4}$ After the report of Witkin $e t a \bar{l}$ of a link between criminality and IQ in $\mathrm{XYY}$ males, others have examined the association between supernumerary $\mathrm{X}$ chromosomes and IQ.

Several prospective cohort studies, begun in the 1970s, of people with SCAs identified at birth reported preliminary findings in the early 1980 s. $^{67}$ The findings suggested that there was impaired performance in both verbal and nonverbal IQ in girls with the XXX genotype and delayed language development in XXY boys. It was hypothesised that there is a continuum of language disability in boys with Klinefelter's syndrome $(\mathrm{XXY})^{6}$ and the idea that an extra sex chromosome may have an adverse effect on neurodevelopment was proposed. ${ }^{8}$

People with Turner's syndrome (XO) show evidence of impaired modular spatial cognition $^{9}{ }^{10}$ and structural neuroimaging studies of SCAs have focused on this condition. ${ }^{11} 12$ Bilateral structural abnormalities of the hippocampi, caudate, lenticular, and thalamic nuclei and parieto-occipital matter, more marked on the right side, have been shown relative to controls. Two functional imaging studies of Turner's syndrome have been performed $^{1314}$ and global hypermetabolism with relative bilateral hypometabolism of the association cortices reported. The inclusion of mosaic subjects in the latter study has lent biological support to the "gene dosing hypothesis" proposed by Murphy et al. ${ }^{14}$

There have, to our knowledge, been no other group structural or functional neuroimaging studies of people with XXX, XYY, or XXY to date. This study examines the hypothesis that the presence of an extra sex chromosome has an effect on neurodevelopment using structural MRI of the brain in people with XXX, XYY, and XXY genotypes.

\section{Methods}

Forty subjects with SCA who participated in the Edinburgh study of growth and development of children were retraced and asked to participate in the current study. The full methodology of this study of consecutive liveborn infants has already been reported. ${ }^{15}$ The study cohort are now aged between 16 and 28 years. Thirty two of the subjects with SCA consented to this study (47 XXX ( $\mathrm{X}=12), 47 \mathrm{XYY}$ $(n=10)$, and $47 \mathrm{XXY}(\mathrm{n}=10))$ and none of these were known to have any concomitant physical disorder affecting brain function. The subjects with SCA were matched groupwise to normal controls (13 female, 26 male, also from the cohort study) of the same phenotypic sex for age, parental social class, and height (within $5 \mathrm{~cm}$ ).

Recent work has shown that none of these subjects fulfil criteria for major psychiatric disorder, although there was an indication that they have an excess of schizoid/schizotypal personality traits. ${ }^{16}{ }^{17}$ The structured interview for schizoptypy $(\mathrm{SIS})^{18}$ was therefore used to measure schizotypal personality traits for this investigation. Global SIS scores were scaled 
Table 1 Demographics: phenotypic females

\begin{tabular}{llll}
\hline & $X X X$ & $X X$ & $p$ Value \\
\hline Number in group & 12 & 13 & \\
Age (mean (SD)) & $21.6(3.1)$ & $21.5(1.4)$ & 1.0 \\
Height in cm (mean(SD)) & $168.8(6.2)$ & $167.7(6.7)$ & 0.7 \\
Median social class & 3 & 3 & 0.8 \\
Right/left handed & $11 / 1$ & $13 / 0$ & 0.5 \\
NART IQ (mean (SD)) & $82.5(7.7)$ & $102.8(7.0)$ & $<0.0001$ \\
Quick IQ (mean (SD)) & $75.6(9.4)$ & $95.0(9.4)$ & $<0.0001$ \\
\hline
\end{tabular}

$1-7$, with a score of 1 as maximal. The SIS, national adult reading test $(\mathrm{NART})^{19}$ of premorbid IQ, and the Quick test ${ }^{20}$ of current IQ were administered to subjects by one investigator (MMW), who was blind to their genotypic status.

Subjects underwent MRI on a 1 tesla Siemens (Erlangen, Germany) Magnetom scanner. Midline sagittal localisation was followed by two sequences to image the whole brain. The first scan was a three dimensional turboFLASH (MPRAGE) sequence consisting of a $180^{\circ}$ inversion pulse followed by a FLASH collection (flip angle $12^{\circ}, \mathrm{TR}=10 \mathrm{~ms}, \mathrm{TE}=4$ $\mathrm{ms}, \mathrm{TI}=200 \mathrm{~ms}$, and relaxation delay time 500 $\mathrm{ms})$. This scan gave 128 contiguous slices, each $1.88 \mathrm{~mm}$ thick. The images were taken in the coronal plane orthogonal to the Talairach plane. The field of view was $250 \mathrm{~mm}$. The second scan was a double spin echo sequence which gave simultaneous proton density and $\mathrm{T} 2$ weighted images $(\mathrm{TR}=3565 \mathrm{~ms}, \mathrm{TE}=20$ and $90 \mathrm{~ms}$ ). Thirty one contiguous $5 \mathrm{~mm}$ slices were acquired in the Talairach plane with a field of view of $250 \mathrm{~mm}$.

Immediately after each subject's scan a large plain test object filled with light oil was scanned in exactly the same place in the coil and in the same orientation as the subject. The test object data was used to correct for inhomogeneity in the radiofrequency coil (because of this the signal fell off at the extremes of the volume scanned in the vertical axis). The mean intensity of a $5 \times 5$ pixel square in the centre slice of the test object data was taken as the optimum intensity. The whole dataset was normalised to this value. By dividing the patient data by the normalised data any deviation from optimum coil response could be corrected.

Image processing was done on Sun $\mathrm{Mi}$ crosystems workstations using the software package "Analyze" (Mayo Foundation, Rochester, MN, USA) to outline and compute neuroanatomical structures. Structures were identified with the assistance of an MRI atlas and volumes were calculated by summing voxels on all brain slices included. Specific regions of interest selected initially were whole brain, right and left $(\mathrm{R}+\mathrm{L})$ pre-frontal lobes, temporal lobes $(\mathrm{R}+\mathrm{L})$, caudate nuclei $(\mathrm{R}+\mathrm{L})$, lentiform nuclei $(\mathrm{R}+\mathrm{L})$, thalamic nuclei $(\mathrm{R}+\mathrm{L})$, amygdalohippocampal complex $(\mathrm{R}+\mathrm{L})$, lateral ventricles $(\mathrm{R}+\mathrm{L})$, third ventricle, and fourth ventricle. The prefrontal, temporal, and amygdalohippocampal volumes were defined according to standard criteria and the remaining regions of interest were outlined by naturalistic boundaries, as in our previous studies. ${ }^{21}$ Asymmetry between groups was assessed for volumes of the lateral ventricles, prefrontal lobes, temporal lobes, and amygdalohippocampal complexes using the formula $\mathrm{R}-\mathrm{L} / \mathrm{R}+\mathrm{L}$.

All image processing was done, blindly, by one investigator (JNK) who compared her results with those of another rater on all brain regions in five brains (Pearson's $r=0.8-1.0$ ). The scans were identified by code number/date of birth only and the investigator performing the analyses was unaware of the status of the subjects.

The second SE scan was examined by a neuroradiologist (JJKB) for any qualitative morphological abnormalities - to exclude gross lesions and to quantify the numbers of high intensity signal (HIS) foci. HIS foci were recorded, if at least $2 \mathrm{~mm}$ in size, by location.

Statistical analysis of results was conducted using SPSS (Microsoft), with separate analyses for the phenotypic females and males. Analysis of variance (ANOVA) with post hoc Scheffe tests (controlling for multiple comparisons) if required were applied to examine group differences in age, height, and IQ. Mann-Whitney $U$ and $\chi^{2}$ tests were used to examine group differences in parental social class, handedness and SIS scores. Group differences in MRI volumes were examined with an ANOVA and post hoc Scheffe tests, controlling for whole brain volume by taking the proportion of each regional volume to whole brain volume. There is, however, some evidence that this approach may not reliably control for whole brain volumes and the volumes of brain regions may also be influenced by body size. We therefore performed analyses of covariance for significantly abnormal brain regions, controlling for whole brain volume and height (as a proxy for body size). Pearson's correlation coefficients were computed to explore the relation between whole brain volumes and IQ as measured by full scale NART and the Quick; Spearman's correlation coefficients to examine associations between SIS scores that differed between the study groups, whole brain volumes, and IQ.

\section{Results}

CLINICAL CHARACTERISTICS

As shown in tables 1 (females) and 2 (males), patients with SCA and controls of the same

Table 2 Demographics: phenotypic males

\begin{tabular}{|c|c|c|c|c|c|}
\hline & $X Y Y$ & $\begin{array}{l}\text { XXY } \\
\text { (Klinefelter) }\end{array}$ & $X Y$ & p Value & Post hoc \\
\hline Number in group & 10 & 10 & 26 & & \\
\hline Age (mean (SD)) & $21.8(3.2)$ & $21.8(4.2)$ & $21.5(1.3)$ & 0.9 & \\
\hline Height in $\mathrm{cm}$ (mean (SD)) & $188.2(4.7)$ & $186.2(4.8)$ & $183.2(4.8)$ & 0.02 & $\mathrm{p}<0.05(\mathrm{XYY}>\mathrm{XY})$ \\
\hline Median social class & 2.5 & 3 & 2 & 0.2 & \\
\hline Right/left handed & $10 / 0$ & $8 / 2$ & $24 / 2$ & 0.3 & \\
\hline NART IQ (mean (SD)) & $90.4(9.3)$ & $86.1(15.8)$ & $105.1(7.6)$ & $<0.001$ & $\mathrm{p}<0.05(\mathrm{XY}>\mathrm{XYY}, \mathrm{XXY})$ \\
\hline Quick IQ (mean (SD)) & $85.0(11.4)$ & $87.2(18.6)$ & $101.2(8.9)$ & 0.001 & $\mathrm{p}<0.05(\mathrm{XY}>\mathrm{XYY}, \mathrm{XXY})$ \\
\hline
\end{tabular}


Table 3 MRI Results: phenotypic females. Group mean (SD) regional brain volumes in $\mathrm{ml}$ are given for information. Proportions of regional to whole brain volume were used for statistical analysis

\begin{tabular}{llll}
\hline & $X X X$ & $X X$ & ANOVA \\
\hline Number scanned & 11 & 13 & \\
Whole brain & $1160.6(66.4)$ & $1251.9(67.7)$ & $\mathrm{F} 1,22=11.0, \mathrm{p}=0.003$ \\
Right prefrontal lobe & $66.5(7.9)$ & $74.9(7.9)$ & $\mathrm{F} 1,22=1.1, \mathrm{p}=0.3$ \\
Left prefrontal lobe & $65.4(10.2)$ & $70.0(9.8)$ & $\mathrm{F} 1,22=0.1, \mathrm{p}=0.8$ \\
Right temporal lobe & $69.1(7.0)$ & $77.1(5.2)$ & $\mathrm{F} 1,22=2.8, \mathrm{p}=0.1$ \\
Left temporal lobe & $66.7(6.8)$ & $73.5(6.6)$ & $\mathrm{F} 1,22=0.4, \mathrm{p}=0.5$ \\
Right caudate nucleus & $3.8(0.4)$ & $4.1(0.5)$ & $\mathrm{F} 1,22=0.004, \mathrm{p}=1.0$ \\
Left caudate nucleus & $3.9(0.4)$ & $4.2(0.5)$ & $\mathrm{F} 1,22=0.1, \mathrm{p}=0.7$ \\
Right lentiform nucleus & $4.2(0.6)$ & $4.6(0.4)$ & $\mathrm{F} 1,22=0.1, \mathrm{p}=0.7$ \\
Left lentiform nucleus & $4.3(0.4)$ & $4.5(0.5)$ & $\mathrm{F} 1,22=0.05, \mathrm{p}=0.8$ \\
Right thalamic nuclei & $5.3(0.7)$ & $5.7(0.6)$ & $\mathrm{F} 1,22=0.01, \mathrm{p}=0.9$ \\
Left thalamic nuclei & $5.6(0.8)$ & $6.2(0.6)$ & $\mathrm{F} 1,22=0.4, \mathrm{p}=0.5$ \\
Right amygdalohippocampal complex & $4.0(0.5)$ & $4.4(0.3)$ & $\mathrm{F} 1,22=0.1, \mathrm{p}=0.7$ \\
Left amygdalohippocampal complex & $3.8(0.3)$ & $4.1(0.4)$ & $\mathrm{F} 1,22=0.02, \mathrm{p}=0.9$ \\
Right lateral ventricle & $3.2(2.3)$ & $4.0(2.7)$ & $\mathrm{F} 1,22=0.3, \mathrm{p}=0.6$ \\
Left lateral ventricle & $4.9(5.9)$ & $3.6(2.2)$ & $\mathrm{F} 1,22=0.7, \mathrm{p}=0.4$ \\
Third ventricle & $0.2(0.1)$ & $0.4(0.2)$ & $\mathrm{F} 1,22=3.7, \mathrm{p}=0.07$ \\
Fourth ventricle & $0.6(0.2)$ & $0.7(0.1)$ & $\mathrm{F} 1,22=0.03, \mathrm{p}=0.9$ \\
\hline
\end{tabular}

phenotypic sex were well matched for age, parental social class, and handedness. Despite matching for height, the XYY group (mean height $188 \mathrm{~cm})$ were significantly $(\mathrm{p}<0.05)$ taller than the XY subjects (mean height 183 $\mathrm{cm}$ ). As would be expected, the IQ scores in all three SCA groups were lower on both the NART and the Quick test than their same phenotypic sex controls, but there were no significant IQ differences between the two male SCA groups (XYY and XXY).

Subjects' SIS scores were dichotomised into those scoring 1, 2, or 3 (high scores) and those subjects scoring $4,5,6$, or 7 (low scores). XXX females were overrepresented, relative to female controls (XX), in the high scoring groups on introversion (Fisher's exact two tailed $\mathrm{p}=0.01$ ), magical thinking (Fisher's $\mathrm{p}=0.04$ ), and impulsivity (Fisher's $\mathrm{p}=0.002$ ). Subjects with XYY were overrepresented in the antisocial traits higher score group compared with normal male controls (Fisher's $p=0.03$ ). There were no significant differences in dichotomised SIS scores between subjects with XXY and normal male controls (XY).

MRI RESULTS

One XXX and one male control were not scanned. As shown in table 3, XXX females had significantly smaller whole brain volumes than female controls. Several other substantial differences in regional volumes are evident, but were not significant after controlling for whole brain volume. To control for the possibility that whole brain volume reductions in $\mathrm{XXX}$ subjects were attributable to general reductions in body size, even though mean height was slightly greater in subjects with $\mathrm{XXX}$, we performed an analysis of covariance of whole brain volume by group and height. This confirmed a significant effect for group $(p=0.002)$ but not for height $(p=0.2)$. There were no significant volumetric asymmetries detected between phenotypic females.

Within the phenotypic males, no significant differences were found between XYY and controls, but several volume differences were found between XXY and controls (table 4). The XXY males had smaller brains than controls, as well as bilaterally enlarged lateral ventricles relative to whole brain volume. The right lateral ventricular volume was also larger in XXY subjects than XYY subjects. Covariance analysis of whole brain findings with group and height confirmed a significant effect for group $(p=0.001)$ but not for height $(p=0.5)$. Similarly, the ventricular findings in XXY subjects remained highly significant after covariance analysis on whole brain volume and height. There were no significant volumetric asymmetries detected between phenotypic males.

One or more HIS foci were detected in three XXX females, but no female controls (Fisher's exact test $\mathrm{p}=0.08$ ). One or more HIS foci were also detected in five XXY subjects $(p=0.001)$ and one XYY subject $(p=0.3)$ whereas none were seen in any male controls.

\section{CORRELATIONS}

Within all female subjects, whole brain volumes were correlated with the full scale NART $(r=0.58, \mathrm{p}=0.004)$ and Quick test $(r=0.53$, $\mathrm{p}=0.008)$ scores. In men, weaker correlations were found with the NART $(r=0.30, p=0.05)$ and the Quick test $(r=0.31, \mathrm{p}=0.04)$. Whole brain and total lateral ventricular volume correlated with Quick IQ scores when all subjects with SCA were analysed as one group $(r=0.38, \mathrm{p}=0.04 ; r=0.62, \mathrm{p}<0.001)$, This was not replicated in normal controls. There were no significant correlations between IQ scores

Table 4 MRI results: phenotypic males. Group mean (SD) regional brain volumes in ml are given for information. Proportions of regional to whole brain volume were used for statistical analysis

\begin{tabular}{|c|c|c|c|c|c|}
\hline & $X Y Y$ & $\begin{array}{l}\text { XXY } \\
\text { (Klinefelter) }\end{array}$ & $X Y$ & ANOVA & Post hoc Scheffe test \\
\hline Number scanned & 10 & 10 & 25 & & \\
\hline Whole brain & $1383.3(99.6)$ & $1296.1(87.8)$ & $1438.0(85.3)$ & $\mathrm{F} 2,42=9.2, \mathrm{p}=0.0005$ & $p \leqslant 0.05(X Y>X X Y)$ \\
\hline Right prefrontal lobe & $79.4(10.5)$ & $76.5(7.8)$ & $83.9(11.3)$ & $\mathrm{F} 2,42=0.2, \mathrm{p}=0.8$ & \\
\hline Left prefrontal lobe & $72.0(6.9)$ & $73.5(7.8)$ & $77.8(11.0)$ & $\mathrm{F} 2,42=1.7, \mathrm{p}=0.2$ & \\
\hline Right temporal lobe & $85.3(9.1)$ & $78.5(6.5)$ & $91.2(5.4)$ & $\mathrm{F} 2,42=3.5, \mathrm{p}=0.04$ & No significant differences \\
\hline Left temporal lobe & $81.4(6.5)$ & $74.8(5.6)$ & $85.2(6.7)$ & $\mathrm{F} 2,42=0.7, \mathrm{p}=0.5$ & \\
\hline Right caudate nucleus & $4.2(0.6)$ & $4.1(0.4)$ & $4.5(0.5)$ & $\mathrm{F} 2,42=0.5, \mathrm{p}=0.6$ & \\
\hline Left caudate nucleus & $4.3(0.6)$ & $4.1(0.5)$ & $4.7(0.5)$ & $\mathrm{F} 2,42=1.0, \mathrm{p}=0.4$ & \\
\hline Right lentiform nucleus & $4.7(0.4)$ & $4.5(0.3)$ & $5.3(0.5)$ & $\mathrm{F} 2,42=1.5, \mathrm{p}=0.2$ & \\
\hline Left lentiform nucleus & $4.9(0.7)$ & $4.5(0.4)$ & $5.3(0.5)$ & $\mathrm{F} 2,42=0.5, \mathrm{p}=0.6$ & \\
\hline Right thalamic nuclei & $6.0(0.6)$ & $5.7(0.5)$ & $6.2(0.8)$ & $\mathrm{F} 2,42=0.1, \mathrm{p}=0.9$ & \\
\hline Left thalamic nuclei & $6.5(0.6)$ & $6.2(0.6)$ & $7.0(0.5)$ & $\mathrm{F} 2,42=0.5, \mathrm{p}=0.6$ & \\
\hline Right amygdalohippocampal complex & $4.9(0.5)$ & $4.4(0.4)$ & $5.0(0.4)$ & $\mathrm{F} 2,42=0.9, \mathrm{p}=0.4$ & \\
\hline Left amygdalohippocampal complex & $4.5(0.5)$ & $4.1(0.3)$ & $4.5(0.4)$ & $\mathrm{F} 2,42=0.4, \mathrm{p}=0.7$ & \\
\hline Right lateral ventricle & $4.6(2.4)$ & $8.1(5.6)$ & $3.6(2.3)$ & $\mathrm{F} 2,42=8.1, \mathrm{p}=0.001$ & $\mathrm{P} \leqslant 0.05(\mathrm{XXY}>\mathrm{XYY}, \mathrm{XY})$ \\
\hline Left lateral ventricle & $5.9(3.2)$ & $7.4(3.5)$ & $4.3(2.9)$ & $\mathrm{F} 2,42=5.8, \mathrm{p}=0.006$ & $p \leqslant 0.05(X X Y>X Y)$ \\
\hline Third ventricle & $0.4(0.2)$ & $0.5(0.3)$ & $0.5(0.3)$ & $\mathrm{F} 2,42=0.9, \mathrm{p}=0.4$ & \\
\hline Fourth ventricle & $1.0(0.3)$ & $1.1(0.6)$ & $1.0(0.5)$ & $\mathrm{F} 2,42=0.8, \mathrm{p}=0.5$ & \\
\hline
\end{tabular}


(either NART or Quick) and whole brain volumes in any individual group (either SCA subjects or normal controls).

None of the three significant different SIS scores in the XXX group (introversion, magical thinking, and impulsivity) correlated with whole brain volume. However, in the normal female control group, impulsivity did show a negative correlation with whole brain volume $(r=-0.57, \mathrm{p}=0.04)$.

In male subjects, antisocial traits were correlated negatively with whole brain volume in the normal control group $(r=-0.52, \mathrm{p}=0.01)$, but not the XYY $(r=0.12, \mathrm{p}=0.7)$, or $\mathrm{XXY}$ groups $(\mathrm{r}=-0.44, \mathrm{p}=0.2)$.

\section{Discussion}

We have found differences in the brain volumes of subjects with SCAs as compared with healthy controls of the same phenotypic sex. The previous careful collection of these consecutively born cases gave us the opportunity to study this unusual population with modern brain imaging and analysis techniques of high resolution. Our main result, of significantly reduced brain volumes in subjects with $\mathrm{XXX}$ and $\mathrm{XXY}$, but not in XYY subjects, indicates that the presence of a supernumerary $\mathrm{X}$ chromosome has a demonstrable effect on brain development.

The number of subjects with SCAs is small, but it is one of the largest known cohorts of such subjects. Moreover, the effects we detected are substantial and are not affected by subject ascertainment bias. We do not have a measure of total intracranial volume as a control reference for whole brain volumes; it is therefore possible that the brain volume reductions are attributable to general reductions in skull or body size. However, there is no one correct method of controlling for these possible influences ${ }^{22-24}$ and covariance analysis for height is generally regarded as a satisfactory way of controlling for body size. ${ }^{23}$ Moreover, the fact that subjects with SCAs were generally taller than their same sex controls supports our conclusion that the brain itself is significantly smaller in subjects with SCAs than healthy controls. It should also be noted that subjects with XXY had enlarged lateral ventricles whether controlling for whole brain volume as a proportion or by covariance.

The correlations between whole brain volume and IQ seen in both the SCA cohort and phenotypic females, suggest that an association between brain size and IQ does exist in these two groups. Others have reported similar findings in normal subjects, especially females ${ }^{25}$ and patients with schizophrenia. ${ }^{21}$ Presumably, the developmentally small brains in subjects with SCAs influence their "premorbid" and current intelligence level, as measured by the NART and Quick tests. There are, of course, several environmental influences on brain size and IQ, such as social class and lifestyle factors. Our cases were matched for paternal social class and this is, therefore, not a probable explanation of our findings.

Our findings of smaller brains and more HIS foci in subjects with SCAs also have more gen- eral neurodevelopmental relevance. Recent imaging studies have shown that X linked conditions (for example, fragile $\mathrm{X}$ syndrome, $\mathrm{X}$ linked pyruvate dehydrogenase deficiency) influence brain development. ${ }^{26}{ }^{27}$ Furthermore, $\mathrm{X}$ linked subcortical laminar heterotopia and lissencephaly syndrome is a neuronal migration disorder manifested by abnormal configuration of the six layers of the neocortex. It has recently been linked to mutations of a novel CNS gene which is highly expressed during brain development in fetal neurons. ${ }^{28}$ The importance of the $\mathrm{X}$ chromosome in ensuring normal fetal neurodevelopment is now well established. Indeed, the total absence of an X chromosome, as in Turner's syndrome, is also associated with brain abnormalities. ${ }^{11}{ }^{12}$ We have shown that the presence of a supernumerary $\mathrm{X}$ chromosome also seems to adversely affect brain development. The finding that the presence of an extra Y chromosome was not associated with significant volumetric brain differences relative to normal male controls may partly reflect low power, but any such effect is at the very least not as dramatic as an extra $\mathrm{X}$. The excess of HIS foci in subjects with a supernumerary X chromosome is of uncertain pathological relevance. Generally HIS foci are thought to reflect the effects of age and any head trauma. ${ }^{29}$ Their presence in XXX and XXY subjects suggests that a developmentally abnormal brain may be more sensitive to such possible insults, or that subjects with SCA s may have an excess of unreported minor head injuries relative to the normal controls.

The correlations between the SIS and brain volumes also suggest that reduced brain volumes may have a role in predisposing to certain behaviours. Some of these could even be mediated by low IQ. However, these analyses were exploratory, could have generated chance findings, and cannot specify a particular causal pathway.

These findings resemble those found in schizophrenia and other neuropsychiatric disorders of presumed developmental origin. ${ }^{30}$ Quantitative reviews of the literature have reported that patients with schizophrenia have substantially reduced whole brain volumes and enlarged lateral ventricles compared with healthy controls. ${ }^{31}{ }^{32}$ Further, there seems to be an excess of XXX and XXY genotypes in people with schizophrenia. ${ }^{33}$ Longitudinal studies of children with supernumerary $\mathrm{X}$ chromosomes have found language deficits, with relative preservation of spatial skills, to be present in some cases. Studies of neurodevelopment in schizophrenia have identified language and motor deficits in childhood before the onset of psychosis in some cases. ${ }^{34-36}$ It has also been reported that people with a diagnosis of mild learning disability have a higher point prevalence (about 3\%) of schizophrenia than the normal population. ${ }^{37}$

Finally, as described above, at the time of scanning none of the subjects in this study fulfilled diagnostic criteria for major mental illness, but they are just entering the period of risk for such disorders. The subjects with SCAs in this study do have more evidence of 
behavioural disturbance and features of asociality $^{16}{ }^{17}$ and one subject, who will be the subject of a separate report, developed a psychosis subsequent to being scanned. It remains to be seen whether more subjects will develop psychosis and whether this may be related to SIS or MRI findings.

MMW was the principal investigator and wrote the first draft of the paper. GAD and SML conducted the data analysis. JNK conducted the quantitative MRI analysis, supervised by SML. JJKB devised the imaging protocols and conducted the qualitaJJKB devised the imaging protocols and conducted the qualita-
tive MRI analysis. ECI designed the study and supervised it tive MRI analysis. ECJ designed the study and supervised it
throughout. All the authors commented on drafts of the paper. throughout. All the authors commented on drafts of the paper. This work was supported by the Wellcome Trust; MMW was a
Wellcome research registrar. We are grateful to Dr Shirley RatWellcome research registrar. We are grateful to Dr Shirley Rat-
cliffe for giving us access to this most interesting cohort and cliffe for giving us access to this most interesting cohort and enriching us with her knowledge of the subject and the individuals, to Annette Blane and Jill Rollo for conducting the developing MRI techniques, to Dr Robert Sandler for assistance in matching subjects, to Dr Anne Hodges for assistance with the administration of the SIS and to Miranda Maxwell and Catherine McLeod for supervising the subjects in the MRI scanner. Finally, we thank all the people who took part in the study.

1 Jacobs PA, Brunton M, Melville MM. Aggressive behaviour, mental subnormality, and the XYY male. Nature 1965;208:1351-2

2 Price WH, Brunton M, Buckton K, et al. Chromosome survey of new patients admitted to the four maximum security hospitals in the United Kingdom. Clin Genet 1976;9:38998.

3 Hunter H. XYY males. Some clinical and psychiatric aspects deriving from a survey of 1811 males in hospitals
for the mentally handicapped. Br $\mathcal{f}$ Psychiatry $1977 ; 131$ : for the $468-77$.

4 Dorus E, Dorus W, Telfer MA, et al. Height and personality characteristics of $47 \mathrm{XYY}$ males in a sample of tall non-institutionalised males. Br F Psychiatry 1976;129:56473.

5 Witkin HA, Mednick SA, Schulsinger F, et al. Criminality in $\mathrm{XYY}$ and XXY men. The elevated crime rate is not related to aggression. It may be related to low intelligence. Science 1976;193:547-55

6 Walzer S, Graham JM Jr, Bashir AS, et al. Preliminary observations on language and learning in XXY boys. Birth Defects 1982;18:185-92

7 Leonard MF, Sparrow S, Schowalter JE. A prospective study of development of children with sex chromosome anomalies-New Haven study III. The middle years. Birth Defects 1982;18:193-218.

8 Theilgaard A. A psychological study of the personalities of XYY and XXY men. Acta Psychiatr Scand 1984; (suppl 315): $1-133$.

9 Murphy DG, Allen G, Haxby JV, et al. The effects of sex steroids, and the $\mathrm{X}$ chromosome, on female brain function: a study of the neuropsychology of adult
syndrome. Neuropsychologia 1994;32:1309-23.

10 Temple CM, Carney RA. Patterns of spatial functioning in Turner's syndrome. Cortex 1995;31:109-18

11 Murphy DGM, DeCarli C, Daly E, et al. X-chromosome effects on female brain: a magnetic resonance imagin study of Turner's syndrome. Lancet 1993;342:1197-200.

12 Reiss AL, Mazzocco MM, Greenlaw R, et al. Neurodevelopmental effects of X monosomy: a volumetric imaging study. Ann Neurol 1995;38:731-8.

13 Clark C, Klonoff H, Hayden M. Regional cerebral glucose metabolism in Turner's syndrome. Can F Neurol Sci 1990; 17:140-4.
14 Murphy DGM, Mentis MJ, Pietrini P, et al. A PET Study of Turner's syndrome: effects of sex steroids and the X chromosome on brain. Biol Psychiatry 1997;41:285-98.

15 Ratcliffe SG, Murray L, Teague P. Edinburgh study of growth and development of children with sex chromosome abnormalities III. Birth Defects 1986;22:73-118.

16 Gotz MJ. The psychiatric consequences of sex chromosome abnormalities: a cohort study [M Phil thesis]. Edinburgh: The University of Edinburgh Library, 1996.

17 Gotz MJ, Johnstone EC, Ratcliffe SG. Criminality and antisocial behaviour in unselected men with sex chromosome abnormalities. Unpublished data.

18 Kendler KS, Lieberman JA, Walsh D. The structured interview for schizotypy (SIS): a preliminary report. Schizophr Bull 1989;15:559-71.

19 Nelson HE, O'Connell A. Dementia; the estimation of premorbid intelligence using the new adult reading test. Cortex 1978;14:234-44.

20 Ammons RB, Ammons CH. Quick IQ test. Missoula: Psychological Test Specialists, 1962 .

21 Lawrie SM, Ingle GT, Santosh CG, et al. Magnetic resonance imaging and single photon emission tomography in treatment-responsive and treatment-resistant schizophrenia. Br F Psychiatry 1995; 167:202-10.

22 Gould SJ. The mismeasure of man. New York, WW Norton, 1981.

23 Arndt S, Cohen G, Alliger RJ, et al. Problems with ratio and proportion measures of imaged cerebral structures. Psychiatry Res 1991;40:79-89.

24 Mathalon DH, Sullivan EV, Rawles JM, et al. Correction for head size in brain-imaging measurements. Psychiatry Res 1993;50:121-39.

25 Andreasen NC, Flaum M, Swayze II V, et al. Intelligence and brain structure in normal individuals. Am $\mathcal{F}$ Psychiatry 1993;150:130-4.

26 Reiss AL, Abrams MT, Greenlaw R, et al. Neurodevelopmental effects of the FMR-1 full mutation in humans. Nature Med 1995;1:159-67.

27 Shevell MI, Matthews PM, Scriver CR, et al. Cerebral dysgenesis and lactic acidaemia: an MRI/MRS phenotype associated with pyruvate dehydrogenase deficiency. Ped associated with pyruvat

28 des Portes V, Pinard, JM, Billuart P, et al. A novel CNS gene required for neuronal migration and involved in $\mathrm{X}$-linked subcortical laminar heterotopia and lissencephaly syndrome. Cell 1998;92:51-61.

29 Munoz DG, Hastak SM, Harper B, et al. Pathological correlates of increased signals of the centrum ovale on magnetic resonance imaging. Arch Neurol 1993;50:492-7.

30 Weinberger DR. From neuropathology to neurodevelopment. Lancet 1995;346:552-7.

31 Ward KE, Friedman L, Wise A, Schulz SC. Meta-analysis of brain and cranial size in schizophrenia. Schizophr Res 1996; 22:197-213.

32 Lawrie SM, Abukmeil SS. How abnormal is the brain in schizophrenia? A systematic and quantitative review of volumetric MRI studies. Br F Psychiatry 1998;172:110-20.

33 DeLisi LE, Friedrich U, Wahistrom J, et al. Schizophrenia and sex chromosome anomalies. Schizophr Bull 1994;20: 495-505.

34 Done DJ, Crow TJ, Johnstone EC, et al. Childhood antecedants of schizophrenia and affective illness: social adjustment at ages 7 and 11. BMF 1994;309:699-703.

35 Walker EF. Neurdevelopmental precursors of schizophrenia. In: David AS, Cutting JC, eds. The neuropsychology of schizophrenia. Hove: Lawrence Ehrlbaum, 1994.

36 Jones P, Rodgers B, Murray R, et al. Child developmental risk factors for adult schizophrenia in the British 1946 birth cohort. Lancet 1994;344:1398-402.

37 Turner TH. Schizophrenia and mental handicap: a historical review with implications for further research. Psychol Med 1989;19:301-14. 